\title{
P I6-02. Induction of systemic HIV-I specific cellular immune responses by oral exposure in exposed uninfected partner of discordant couples
}

\author{
C Pérez ${ }^{* 1}$, K Hasselrot ${ }^{2}$, K Broliden² and A Karlsson ${ }^{3}$
}

Address: ${ }^{1}$ MTC, Karolinska Insitutet, Solna, Sweden, ${ }^{2}$ Center for Molecular Medicine, Karolinska Institutet, Solna, Sweden and ${ }^{3}$ Karolinska Institute, Swedish Institute of Infectious Disease Control, Solna, Sweden

* Corresponding author

from AIDS Vaccine 2009

Paris, France. 19-22 October 2009

Published: 22 October 2009

Retrovirology 2009, 6(Suppl 3):P23I doi:I0.II86/I742-4690-6-S3-P23I

This abstract is available from: http://www.retrovirology.com/content/6/S3/P23।

(c) 2009 Pérez et al; licensee BioMed Central Ltd.

\section{Background}

No protective immune response has been identified so far and no HIV-vaccine has yet proven successful. However, some individuals seem to be naturally protected against HIV, or at least less susceptible for HIV transmission. These so called exposed uninfected individuals have been heavily studied in hope to revealing the mechanisms behind the protection. Such information would be highly relevant for any HIV-vaccine design. Our aim was to investigate whether oral exposure of HIV is enough to induce a systemic HIV-1 specific cellular immune response.

\section{Methods}

25 EU homosexual men living with a HIV-positive partner were included in the study. The discordant couples had unprotected oral sex, and only rarely unprotected anal intercourse. Detection of HIV-1 specific cellular responses was performed using the IFN- $\gamma$ ELISPOT assay and stimulated with five consensus $B$ peptide pools. Identification of production of several cytokines and determination of HIV-specific CD4+ and CD8+ producing T-cells were made by 8-colour flow based Intracellular Cytokine Staining (ICS).

\section{Results}

3 out of the 25 (12\%) study subjects had HIV specific cellular immune responses. One had strong Gag-p55 and Gag-p24 peptide pool responses, and two subjects responded against the HIV-Nef peptide pool. The Gag- responder had multiple HIV-specific cytokine production in both CD4+ and CD8+ T-cells. The magnitude of the responses decreased over time, but was still detectable in the CD4+ T-cells one year after HIV-exposure had decline.

\section{Conclusion}

We show that oral exposure is sufficient to induce systemic HIV-1 specific cellular responses. This is the first time HIV-specific $\mathrm{T}$ cell responses have been detected in exposed uninfected individuals with oral HIV-exposure. Whether these cellular responses have any protective role against HIV infection, or if they rather are nothing but a foot print of exposure, needs to be further investigated. 